This item was submitted to Loughborough's Research Repository by the author.

Items in Figshare are protected by copyright, with all rights reserved, unless otherwise indicated.

\title{
Hot electron relaxation in the heavy-fermion Yb1-xLuxAl3 compound using femtosecond optical pump-probe spectroscopy
}

PLEASE CITE THE PUBLISHED VERSION

\section{PUBLISHER}

(C) American Physical Society

\section{VERSION}

VoR (Version of Record)

\section{LICENCE}

CC BY-NC-ND 4.0

\section{REPOSITORY RECORD}

Demsar, J., V.V. Kabanov, A.S. Alexandrov, H.J. Lee, E.D. Bauer, J.L. Sarrao, and A.J. Taylor. 2019. "Hot Electron Relaxation in the Heavy-fermion Yb1-xluxal3 Compound Using Femtosecond Optical Pump-probe Spectroscopy". figshare. https://hdl.handle.net/2134/5845. 
This item was submitted to Loughborough's Institutional Repository (https://dspace.lboro.ac.uk/) by the author and is made available under the following Creative Commons Licence conditions.

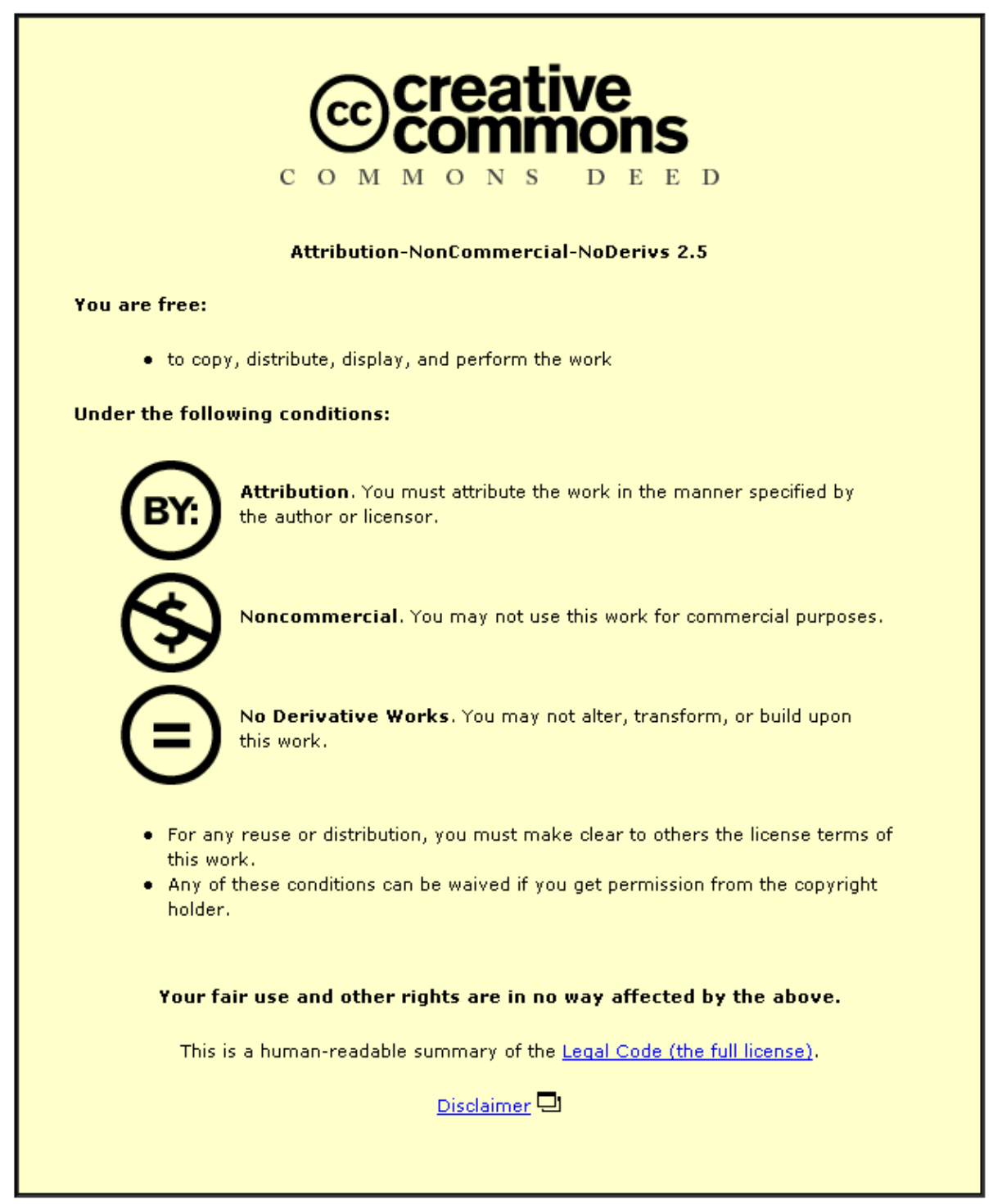

For the full text of this licence, please go to: http://creativecommons.org/licenses/by-nc-nd/2.5/ 


\title{
Hot electron relaxation in the heavy-fermion $\mathrm{Yb}_{1-x} \mathrm{Lu}_{x} \mathrm{Al}_{3}$ compound using femtosecond optical pump-probe spectroscopy
}

\author{
J. Demsar, ${ }^{1,2,3}$ V. V. Kabanov,,${ }^{1,3}$ A. S. Alexandrov,${ }^{4}$ H. J. Lee, ${ }^{5}$ E. D. Bauer, ${ }^{5}$ J. L. Sarrao, ${ }^{5}$ and A. J. Taylor ${ }^{5}$ \\ ${ }^{1}$ Physics Department and Zukunftskolleg, University of Konstanz, D-78457 Konstanz, Germany \\ ${ }^{2}$ Center for Applied Photonics, University of Konstanz, D-78457 Konstanz, Germany \\ ${ }^{3}$ Complex Matter Department, Jozef Stefan Institute, SI-1000 Ljubljana, Slovenia \\ ${ }^{4}$ Department of Physics, Loughborough University, Loughborough LE11 3TU, United Kingdom \\ ${ }^{5}$ Los Alamos National Laboratory, Los Alamos, New Mexico 87545, USA
}

(Received 4 August 2009; published 27 August 2009)

\begin{abstract}
Femtosecond time-resolved optical spectroscopy was used to systematically study photoexcited carrier relaxation dynamics in the intermediate-valence heavy-fermion system $\mathrm{Yb}_{1-x} \mathrm{Lu}_{x} \mathrm{Al}_{3}(0 \leq x \leq 1)$. Given the demonstrated sensitivity of this experimental technique to the presence of the low-energy gaps in the charge excitation spectrum, the aim of this work was to study the effect of dilution of the Kondo lattice on its low-energy electronic structure. The results imply that in $\mathrm{Yb}_{1-x} \mathrm{Lu}_{x} \mathrm{Al}_{3}$ the hybridization gap, resulting from hybridization of local moments and conduction electrons, persists up to $30 \%$ doping. Interestingly, below some characteristic, doping dependent temperature $T^{*}(x)$ the relaxation-time divergence, governed by the relaxation bottleneck due to the presence of the indirect hybridization gap, is truncated. This observation is attributed to the competing ballistic transport of hot electrons out of the probed volume at low temperatures. The derived theoretical model accounts for both the functional form of relaxation dynamics below $T^{*}(x)$, as well as the doping dependence of the low-temperature relaxation rate in $\mathrm{Yb}_{1-x} \mathrm{Lu}_{x} \mathrm{Al}_{3}$.
\end{abstract}

DOI: 10.1103/PhysRevB.80.085121

PACS number(s): 78.47.- p, 71.27.+a, 71.28.+d, 78.55. $-\mathrm{m}$

\section{INTRODUCTION}

A reasonable understanding of the Kondo problem was achieved in the case of isolated Kondo impurities by the Anderson impurity model (AIM). Despite the fact that many heavy electron systems are stoichiometric with a local moment in each unit cell, many of their physical properties can be well accounted for by the AIM. On the other hand, some low-temperature properties such as optical conductivity are fundamentally different than those expected of the AIM. These are consistent with the renormalized band behavior, captured by the Anderson lattice model. Here hybridization of local moments and conduction electrons leads to the presence of the hybridization gap (HG) in the vicinity of the Fermi level, ${ }^{1-3}$ where in heavy fermions the Fermi level lies close to the edge of the lower hybridized band. ${ }^{4,5}$

Recent experiments on the photoexcited carrier relaxation dynamics in several heavy electron compounds have shown that the relaxation of the electronic system back to equilibrium is extremely sensitive to the underlying low-energy electronic structure and strongly depends on the temperature (T) and excitation level. ${ }^{4-8}$ It has been shown, that the bottleneck in carrier relaxation is governed by the presence of the HG near the Fermi level. ${ }^{4,5}$ Both $T$ and excitation density dependence of the relaxation process can be well accounted for by the phenomenological Rothwarf-Taylor (RT) model,,${ }^{5} 9-11$ which was originally developed to describe the relaxation in superconductors driven out of equilibrium. ${ }^{9-11}$ We should note that the alternative model presented in Refs. 6 and 7 is essentially also a bottleneck model. Here the energy and momentum conservation law leads to the suppression of electron-phonon (e-ph) scattering near $E_{f}$ in the case of sound velocity exceeding the Fermi velocity. This suppression of e-ph scattering near $E_{f}$ effectively acts as a gap in the density of states (DOS). However, since the temperature and excitation dependence of the relaxation dynamics in a Kondo insulator $\mathrm{SmB}_{6}$ is nearly identical to that of heavyfermion systems, ${ }^{4,5}$ it was naturally to assume that the origin of the bottleneck in the entire class of materials was in the presence of the indirect hybridization gap. ${ }^{4,5}$

In this paper we report first systematic studies of photoexcited carrier relaxation dynamics in an intermediatevalence system $\mathrm{Yb}_{1-x} \mathrm{Lu}_{x} \mathrm{Al}_{3}$. In $\mathrm{Yb}_{1-x} \mathrm{Lu}_{x} \mathrm{Al}_{3}$ concentration of local $f$ moments can be continuously varied between 1 $\left(\mathrm{YbAl}_{3}\right.$ is a heavy fermion with the Kondo temperature of $600-700 \mathrm{~K}$ ) and 0 by replacing $\mathrm{Yb}$ with open $f$-shell with $\mathrm{Lu}$ with closed $f$ shell ( $\mathrm{LuAl}_{3}$ is a normal metal). Dilution by doping with nonmagnetic ions should give rise to the disappearance of the long-range order, and the associated $\mathrm{HG}$ at some critical doping. Moreover, in $\mathrm{YbAl}_{3}$ the lowtemperature anomalies observed in susceptibility and specific heat suggest the presence of a second energy scale of the order of $40 \mathrm{~K} .{ }^{12}$ The observed anomalies are quickly suppressed by doping ${ }^{13}$ suggesting that this energy scale is related to the onset of Fermi-liquid coherence. ${ }^{12,13}$ Using optical pump-probe spectroscopy in the low perturbation regime we show that at high $T$ and doping levels $0 \leq x \leq 0.3$ the relaxation rate decreases with decreasing $T$ much like in other heavy electron systems studied so far. ${ }^{4-6}$ This behavior is consistent with the relaxation bottleneck due to the presence of the HG. ${ }^{4}$ The HG does not change significantly in the doping range $0 \leq x \leq 0.3$, and the disappearance of the longrange order appears near $x \approx 0.4$.

At some characteristic doping dependent temperature $T^{*}(x)$, the slowing down of relaxation is truncated and the relaxation rate becomes constant below $T^{*}$. This behavior is explained by the competition between the relaxation across the HG and the ballistic electron transport, when at low $T$ the 

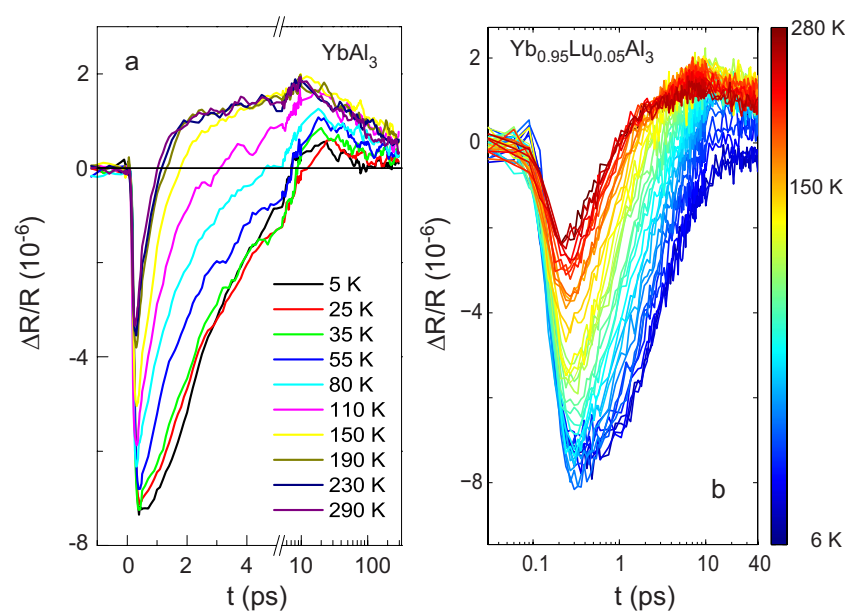

FIG. 1. (Color online) Photoinduced reflectivity traces in (a) $\mathrm{YbAl}_{3}$ at selected temperatures and in (b) $\mathrm{Yb}_{0.95} \mathrm{Lu}_{0.05} \mathrm{Al}_{3}$ from $6-280 \mathrm{~K}$ (in $6 \mathrm{~K}$ steps between $6-140 \mathrm{~K}$ and $10 \mathrm{~K}$ steps from 140-280 K).

electron mean-free path $l$ becomes larger than the optical penetration depth $\lambda$. We derived the theoretical model that describes the functional form of relaxation for such a case and discuss the striking doping dependence of the lowtemperature relaxation rate.

\section{EXPERIMENTAL}

The experiments were performed in the standard pumpprobe configuration using a mode-locked Ti:sapphire laser as the source for both photoexcitation and probe-laser-pulse trains. The pump and probe pulses had a duration of $<20 \mathrm{fs}$ at the center wavelength of $800 \mathrm{~nm}$. The experiments were performed in the very weak excitation regime with the excitation fluence of $F \approx 0.4 \mu \mathrm{J} / \mathrm{cm}^{2}$. ${ }^{14}$ From the literature values ${ }^{15}$ of the complex conductivity at $1.5 \mathrm{eV}$ we estimated the corresponding absorbed energy densities to be $\approx 90 \mathrm{~mJ} / \mathrm{cm}^{3}$ in $\mathrm{YbAl}_{3}$. Based on the known values of the Sommerfeld constant we estimated the corresponding increase in the electronic temperature after excitation to be less than $10 \mathrm{~K}$ over the entire $T$ range implying that the dynamics being studied is near the thermal equilibrium. The $\mathrm{Yb}_{1-x} \mathrm{Lu}_{x} \mathrm{Al}_{3}$ samples (ten doping levels were studied) were grown by the "self-flux" method in excess Al. ${ }^{13}$

\section{RESULTS}

Figure 1 shows the dynamics of photoinduced reflectivity change in $\mathrm{YbAl}_{3}$ and $\mathrm{Yb}_{0.95} \mathrm{Lu}_{0.05} \mathrm{Al}_{3}$ in the $T$ range between 4 and $300 \mathrm{~K}$. At $300 \mathrm{~K}$, the rise time of about $70 \mathrm{fs}$ is followed by fast electronic relaxation which can be well described by a single exponential decay with the relaxation time $\tau=330$ fs. Following this initial electronic relaxation the system recovers to equilibrium on the time scale of several $100 \mathrm{ps}$, which is attributed to heat flow out of the excitation volume. Upon lowering $T$, changes in both the amplitude of the transient, $\mathbf{A}$, and $\tau$ are observed. Upon cooling $\tau$ gradually increases in the same fashion as in previously stud-

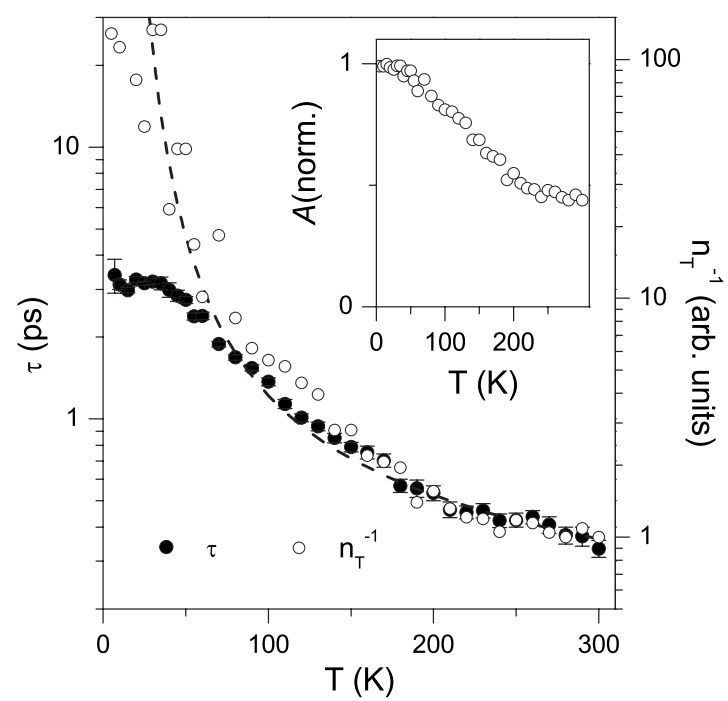

FIG. 2. $T$ dependence of $\tau$ and the inverse density of thermally excited quasiparticles, $n_{T}^{-1}$, for $\mathrm{YbAl}_{3} \cdot n_{T}^{-1}$ was determined from the $T$ dependence of the normalized amplitude of the photoinduced reflectivity change, $\mathcal{A}$ (inset), via $n_{T} \propto \mathcal{A}^{-1}-1$. The data are fit with $n_{T} \simeq T^{1 / 2} \exp \left(-E_{h g} / 2 T\right)$, where $E_{h g}=16 \mathrm{meV}$ is the size of the indirect HG.

ied heavy electron compounds, ${ }^{4-6}$ reaching $3.2 \mathrm{ps}$ at $\approx 50 \mathrm{~K}$. There the anomalous increase in $\tau$ is truncated and $\tau$ remains constant below $\approx 50 \mathrm{~K}$. The amplitude of transient, $\mathbf{A}$, also shows a pronounced $T$ dependence similar to early reports. ${ }^{4}$ At low temperatures $\mathbf{A}$ is being nearly constant up to $\approx 50 \mathrm{~K}$ and showing a pronounced suppression upon further $T$ increase. Qualitatively similar results are obtained for all 0 $<x<0.3$, except that the temperature where the relaxationtime divergence is truncated, $T^{*}$, is doping dependent, as discussed below. In $\mathrm{LuAl}_{3}$, however, the dynamics follows the behavior seen in normal metals, where the relaxation is only very weakly temperature dependent with the relaxation time slightly increasing upon increasing the temperature. ${ }^{16}$ Similarly, virtually no $T$ dependence of $\mathcal{A}$ is observed.

The $T$ dependence of $\tau$ in $\mathrm{YbAl}_{3}$ is shown in Fig. 2 . Above $\approx 50 \mathrm{~K}$ it is consistent with the relaxation bottleneck scenario due to the presence of the narrow $\mathrm{HG}, E_{h g}$, in the density of states. ${ }^{4,5}$ The relaxation process can be described by the RT model. Here following photoexcitation and the initial electron-electron and electron-phonon collisions which proceed on a sub-ps time scale, the system is characterized by excess densities of electron-hole pairs (EHPs) and high-frequency phonons (HFPs). When an EHP with an energy $\geq E_{h g}$ recombines, a high-frequency phonon $\left(\omega>E_{h g}\right)$ is created. Since HFP can subsequently excite EHP, the recovery is essentially governed by the decay of the HFP population. ${ }^{9,11}$ In the low excitation limit, as in this case, the $T$ dependencies of both amplitude of the induced reflectivity change $\mathbf{A}$, and $\tau$ are governed by the $T$ dependence of the number density of thermally excited EHPs, $n_{T}$. It was shown ${ }^{11}$ that $n_{T} \propto \mathcal{A}^{-1}-1$, where $\mathcal{A}(T)=\mathbf{A}(T) / \mathbf{A}(T \rightarrow 0)$ and

$$
\tau(T) \propto\left[\delta \mathcal{A}(T)+2 n_{T}(T)\right]^{-1},
$$

where $\delta$ is a constant that depends only on the photoexcitation intensity. In a narrow-band semiconductor $n_{T}$ depends 
on the shape of the DOS in the energy range $\varepsilon \approx T$ around the chemical potential (here and further $k_{B}=1$ ). Generally, the number density of thermally excited EHPs across the gap (in this case indirect hybridization gap, $E_{h g}$ ) is given by

$$
n_{T} \simeq T^{p} \exp \left(-E_{h g} / 2 T\right)
$$

where $p$ is of the order of 1 ( 0.5 for a BCS superconductor), depending on the shape of the DOS near the gap edge. Neither the size of $E_{h g}$, the exact shape of the low-energy DOS (anisotropy, possible impurity levels within the gap), or the $T$ dependence of $E_{h g}$ is well known in intermediate-valence systems, therefore there is some ambiguity in determining $E_{h g}$. However, as the main $T$ dependence in $n_{T}$ comes from the exponential term, a rough estimate of the size of $E_{h g}$ can be obtained. For $T>50 \mathrm{~K}$ a good agreement of the data with this simple model can be obtained. Since $\tau$ was found to be independent on $F$ over the range of $F$ studied $^{14}$ we can assume that $\tau(T) \approx n_{T}^{-1}$. Indeed Fig. 2 shows that there is a good agreement between $\tau(T)$ and $n_{T}^{-1}(T) \propto\left(\mathcal{A}^{-1}-1\right)^{-1}$. Fit with $n_{T}^{-1} \propto T^{-0.5} \exp \left(E_{h g} / 2 T\right)$ gives a values for the indirect $\mathrm{HG}$ of $E_{h g} \approx 16 \mathrm{meV}$. This value is lower than the values of the pseudogap obtained from the inelastic neutron-scattering data $(30 \mathrm{meV}),{ }^{17}$ as well as the value inferred from the optical conductivity data on $\mathrm{YbAl}_{3}(60 \mathrm{meV}) .{ }^{18}$ The relationship between the observed pseudogaps in the spin (neutrons) and charge (optical) excitation spectra is unclear. On the other hand, the interpretation of the optical conductivity data is also not straightforward ${ }^{18}$ since the indirect transitions are forbidden by the momentum conservation law. Furthermore, the relaxation dynamics bottleneck is expected to be governed by the gap minimum, therefore taking the uncertainties in the interpretation of both experimental results the agreement is reasonable. Given the fact, that the mid infrared peak at $0.25 \mathrm{eV},{ }^{18}$ which is in the intermediate-valence systems commonly interpreted as the direct optical transition across the hybridization gap, ${ }^{2}$ is in $\mathrm{YbAl}_{3}$ present way above room temperature, ${ }^{18}$ it is expected that the same is true also for the indirect gap, as implied by the present study.

Figure 3 presents the $T$ dependence of $\tau$, obtained by the single exponential fit, on a series of $\mathrm{Yb}_{1-x} \mathrm{Lu}_{x} \mathrm{Al}_{3}$ samples. Over a large range of doping, $0 \leq x \leq 0.3$, all $\tau\left(T>T^{*}\right)$ fall almost on the same curve. Since $\tau\left(T>T^{*}\right)$ can be well understood in terms of the RT scenario, ${ }^{4,5}$ the results imply that the HG persists over wide range of doping. We should note that there is some variation in the gap extracted from the fit to the relaxation times of individual doping levels $\left(10<E_{h g}<21 \mathrm{meV}\right.$ ) (see the two fits in Fig. 3). However, there is no systematic dependence of $E_{h g}(x)$. At $x=0.4$, however, a qualitative change in the relaxation dynamics is observed, with relaxation showing a very weak temperature dependence, much like in the metallic $\mathrm{LuAl}_{3}$. This observation can be attributed to the loss of the long-range order at $x=0.4$, consistent with the inelastic neutron-scattering data. ${ }^{17}$ Based on the above we argue that the hybridization gap in $\mathrm{Yb}_{1-x} \mathrm{Lu}_{x} \mathrm{Al}_{3}$ persists, nearly unchanged, up to $x \simeq 0.3$, with the loss of the long-range order to appear only near $x \simeq 0.4$.

Below $T^{*}(x)$, however, $\tau$ saturates at $\tau_{*}(x)$. Generally, the saturation of $\tau$ at low $T$ is expected within the RT model when $n_{T}(T)$ becomes smaller than $\delta \mathcal{A}(T)$ [see Eq. (1)]. How-

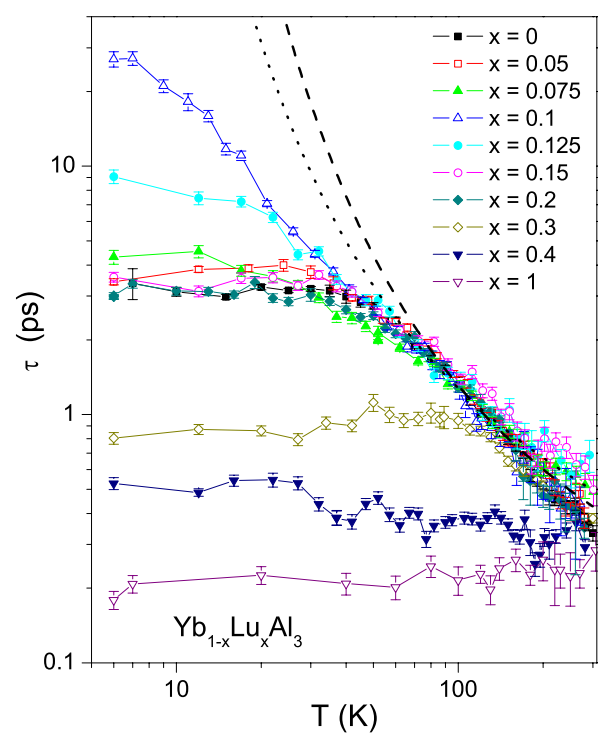

FIG. 3. (Color online) The temperature dependence of $\tau$, extracted using a single exponential decay fit, for $\mathrm{Yb}_{1-x} \mathrm{Lu}_{x} \mathrm{Al}_{3}$ series. The dashed and dotted lines present fits with $\tau \propto T^{-0.5} \exp \left(E_{h g} / 2 T\right)$ with $E_{h g}=17 \mathrm{meV}$ and $E_{h g}=10 \mathrm{meV}$, respectively.

ever in this case the low-temperature relaxation time, $\tau_{*}$, should depend on the excitation fluence. The fact that relaxation dynamics in this low perturbation experiment is found to be independent of excitation intensity effectively rules out this interpretation. ${ }^{14}$ Alternatively, we can assume (quite generally) that the relaxation of the excited state proceeds via two independent channels. In the case of two competing relaxation processes the recovery dynamics will always be governed by the fastest of the two. If one of the processes is slow and temperature independent, while the other shows strong temperature dependence, becoming slower and slower as the temperature is reduced, one would expect to observe a crossover from the temperature dependent to a temperature independent relaxation at the temperature where the two time scales become comparable. Clearly, this scenario is consistent with the experimentally observed temperature dependence of the relaxation time. While at temperatures above $T^{*}$ the temperature dependence of both amplitude and relaxation time are in very good agreement with the relaxation bottleneck model (see Fig. 2), where the bottleneck in relaxation is a result of the presence of the hybridization gap near the Fermi level, the question arises: which temperature independent relaxation process could lead to the observed crossover into $T$-independent relaxation below $T^{*}$ ?

\section{ANALYSIS AND DISCUSSION}

Measurements of the de Haas-van Alphen (dHvA) effect in $\mathrm{YbAl}_{3}$ and $\mathrm{LuAl}_{3}$ indicate a large mean-free path, $l \approx 120-150 \mathrm{~nm},{ }^{19,20}$ several times larger than the optical penetration depth $\lambda\left(\lambda_{\mathrm{YbAl}_{3}}=22 \mathrm{~nm}\right.$ and $\left.\lambda_{\mathrm{LuAl}_{3}}=17 \mathrm{~nm}\right){ }^{15}$ From the $T$ dependence of resistivity (inset to Fig. 5) it follows that $l>\lambda$ up to $\approx 50 \mathrm{~K}$, well into experimentally accessible $T$ range. Hence one can expect that in addition to the recombination process with characteristic time $\tau$ a ballistic 
transport of hot electrons ${ }^{21}$ will take place on a time scale of $\lambda / v_{F}$, where $v_{F}$ is the Fermi velocity. From the dHvA data ${ }^{19,20}$ one obtains $v_{F} \approx 4 \times 10^{4}\left(1 \times 10^{6}\right) \mathrm{m} / \mathrm{s}$ in $\mathrm{YbAl}_{3}\left(\mathrm{LuAl}_{3}\right)$ giving the characteristic time scale for the ballistic transport out of the probed volume of $\lambda / v_{F}=0.5 \mathrm{ps}$ and $20 \mathrm{fs}$, respectively. Since $v_{F}$ is a weak function of temperature, the competition between the strongly $T$ dependent recombination across the $\mathrm{HG}$ and a $T$-independent ballistic transport can account for the observed temperature dependence of the relaxation time in $\mathrm{Yb}_{1-x} \mathrm{Lu}_{x} \mathrm{Al}_{3}$.

To describe the relaxation process for such a case we use the Boltzmann kinetic equation with the collision integral in the $\tau$ approximation, ${ }^{22}$

$$
\frac{\partial f}{\partial t}+v_{F} \cos (\theta) \frac{\partial f}{\partial x}=-\frac{f}{\tau(\epsilon, \theta)} .
$$

Here $f(t, x, \epsilon, \theta)$ is the nonequilibrium correction to the equilibrium distribution function, which depends on time $t$, distance from the surface $x$, energy $\epsilon$ relative to the Fermi energy, and the angle $\theta$ between the velocity and the transport direction, $x$. The relaxation time $\tau$ in general depends on the energy and the angle. Here we assume that the particle-hole symmetry is preserved, so that there is no electric field. Also, if the Fermi energy is large compared to the photon energy, one can neglect the dependence of the speed of hot electrons and holes on their relative energy, i.e., $v \approx v_{F}$. Equation (3), supplemented by the initial condition $f(t=0, x, \epsilon, \theta)=F(\epsilon, x)$, describing the initial distribution of the hot quasiparticles after photoexcitation, has the solution,

$$
f(t, x, \epsilon, \theta)=\exp (-t / \tau(\epsilon, \theta)) F\left(\epsilon, x-v_{F} \cos (\theta, t)\right) .
$$

The spatial and time distribution of the electron-hole pair density is found as

$$
n(x, t)=\int_{0}^{\infty} d \epsilon \int_{0}^{\pi} f(t, x, \epsilon, \theta) \sin (\theta) d \theta .
$$

For comparison with the experimental data we assume that $\tau$ is energy and angle independent, $\tau(\epsilon, \theta)=\tau$ and take the Gaussian form of the energy-integrated excitation profile at $t=0, \int_{0}^{\infty} F(\epsilon, x) d \epsilon=\frac{2}{\sqrt{\pi}} \exp \left(-x^{2} / \lambda^{2}\right) .{ }^{23} \Delta R / R$ is proportional to the number of photoinduced carriers within $\lambda$. Integrating Eq. (5) with the Gaussian probe profile, $\exp \left(-x^{2} / \lambda^{2}\right),{ }^{23}$ and normalizing to 1 at $t=0$ one obtains

$$
\Delta R / R \propto n(t)=\sqrt{\frac{\pi}{2}} \frac{\lambda}{v_{F} t} e^{-t / \tau} \operatorname{erf}\left(v_{F} t / \sqrt{2} \lambda\right) .
$$

Equation (6) has two regimes. On a short time scale, $t \leq \lambda / v_{F}$, it reduces to

$$
n(t)=\exp \left(-t / \tau-v_{F}^{2} t^{2} / 2 \lambda^{2}\right),
$$

while for $t \gg \lambda / v_{F}$ we have

$$
n(t)=\frac{\lambda}{v_{F} t} e^{-t / \tau} .
$$

Figure 4 presents the photoinduced reflectivity traces in $\mathrm{YbAl}_{3}$ at a few selected temperatures. We fit the photoinduced reflectivity traces with Eq. (6) - solid lines and com-

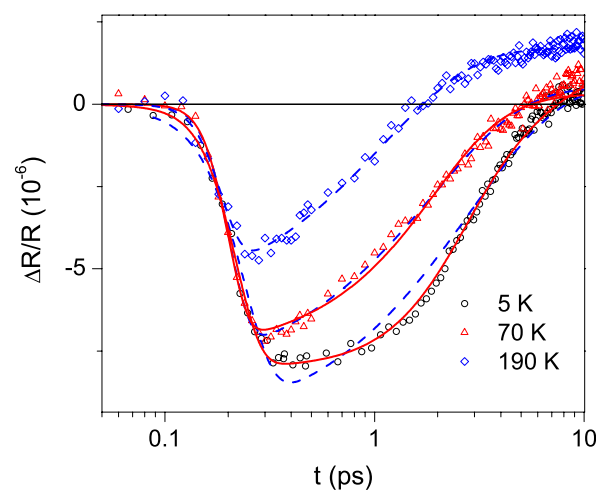

FIG. 4. (Color online) The data recorded on $\mathrm{YbAl}_{3}$ at selected temperatures fit with single exponential decay (dashed blue line) and with Eq. (6) (solid red line), where $\lambda / v_{F}=1.33$ ps. At low temperatures the fit is entirely governed by the $\lambda / v_{F}$ term (with $\tau>5 \mathrm{ps}$ ), while above $50 \mathrm{~K}$ the relaxation is best fit with a single exponential decay.

pare it to the single exponential decay (dashed).

At $T<T^{*}$ the model clearly describes the experimental data much better than a single exponential decay. In fact, the fit is mainly governed by the $\lambda / v_{F}$ ratio (best fit gives $\lambda / v_{F}=1.33 \pm 0.05 \mathrm{ps}$ in good agreement with the above estimate) while $\tau$ can be anywhere between 5-100 ps. The situation is reversed at $T>T^{*}$, where the fit is entirely governed by $\tau$. Since the mean-free path quickly decreases below $\lambda$ upon increasing $T$, e.g., in $\mathrm{YbAl}_{3}$ this should happen at $\approx 50 \mathrm{~K}$ (see inset to Fig. 5), the ballistic transport becomes ineffective. Correspondingly, the fit to the $70 \mathrm{~K}$ data in Fig. 4 with fixed $\lambda / v_{F}=1.33$ clearly becomes inadequate. In fact, the data are much better fit with the single exponential decay (dashed blue line). Thus, $T^{*}$ can be thought of as the temperature where $l \approx \lambda$; below $T^{*}$ the relaxation is dominated by the ballistic transport, while above this temperature relaxation is governed by the recombination across the indirect HG. In earlier studies ${ }^{5}$ such a saturation of $\tau$ at low $T$ was not observed. This can be understood, since at experimentally accessible temperatures of $T \gtrsim 10 \mathrm{~K}$ the mean-free path in $\mathrm{YbAl}_{3}$ is by far the largest among the systems studied. Still,

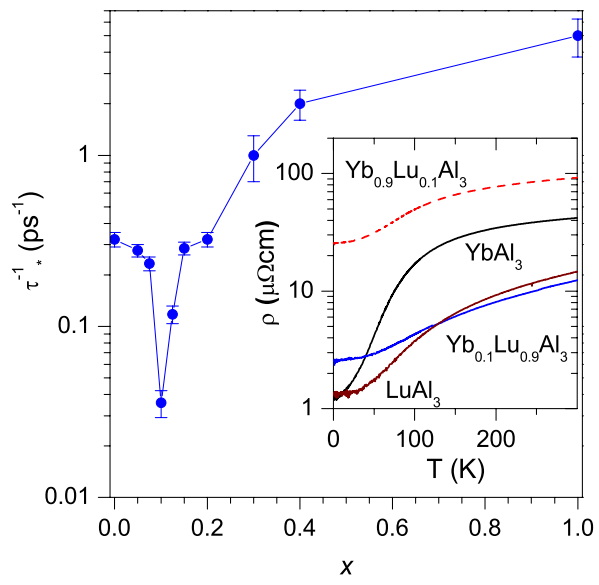

FIG. 5. (Color online) Doping dependence of $\tau_{*}^{-1}$ for $\mathrm{Yb}_{1-x} \mathrm{Lu}_{x} \mathrm{Al}_{3}$. Inset shows the $T$ dependence of resistivity for several doping levels. 
comparison of dynamics in $\mathrm{YbCdCu}_{4}$ and $\mathrm{YbAgCu}_{4}$ (Fig. 5 of Ref. 4) shows an onset of saturation at $\approx 20 \mathrm{~K}$ in $\mathrm{YbAgCu}_{4}$. This feature is absent in $\mathrm{YbCdCu}_{4}$. Since the mean-free path in $\mathrm{YbCdCu}_{4}$ is considerably smaller than in $\mathrm{YbAgCu}_{4},{ }^{24}$ this observation is consistent with the proposed scenario.

The doping dependence of the low-temperature relaxation rate, $\tau_{*}^{-1}(x)$, is plotted in Fig. 5. Initially $\tau_{*}^{-1}$ shows a weak decrease from $\tau_{*}^{-1}(x=0) \approx 0.2 \mathrm{ps}^{-1}$, followed by a rapid drop at $x=0.1$. Further increase in $x$ results in an increase in $\tau_{*}^{-1}$ up to $\approx 5 \mathrm{ps}^{-1}$. Within the above scenario, following the $\lambda / v_{F}$ ratio, one would expect $\tau_{*}^{-1}\left(\mathrm{LuAl}_{3}\right) \approx 20 \tau_{*}^{-1}\left(\mathrm{YbAl}_{3}\right)$, as observed. The strong drop in $\tau_{*}^{-1}$ near $x=0.1$ can be understood as being a result of the decrease of $l$ below $\lambda$, consistent with the resistivity data (see insert to Fig. 5). Indeed, in $\mathrm{Yb}_{0.9} \mathrm{Lu}_{0.1} \mathrm{Al}_{3}$ the expected low- $T$ divergence of $\tau$ due to the relaxation bottleneck ${ }^{4,5}$ is largely recovered (see Fig. 3). Thus, the qualitative doping dependence of $\tau_{*}^{-1}$ in $\mathrm{Yb}_{1-x} \mathrm{Lu}_{x} \mathrm{Al}_{3}$ can be understood as a competition between decreasing $\lambda / v_{F}$ ratio and doping induced disorder. Still, the underlying origin of the very sharp anomaly at $x \approx 0.1$ is unclear. Noteworthy, this doping level corresponds well with the doping level where low-temperature coherence effects are strongly suppressed. ${ }^{12,13}$

\section{CONCLUSIONS}

We studied the carrier relaxation dynamics in $\mathrm{Yb}_{1-x} \mathrm{Lu}_{x} \mathrm{Al}_{3}$, an intermediate-valence heavy-fermion system where doping was continuously tuned between a heavy fermion and normal metal. In $\mathrm{YbAl}_{3}$ there seems to be an overall agreement that its low-temperature properties are governed by a hybridization of conduction electrons with spatially extended wave functions and localized $f$ orbitals, which can be described by the Anderson lattice model. Since photoexcited carrier relaxation was found to be very sensitive to the appearance of the gap in the charge excitation spectrum, the aim of this work was to utilize time-resolved optical spectroscopy to determine the doping level at which long-range order, leading to the indirect hybridization gap in intermediate-valence heavy electron systems, is suppressed. By performing systematic studies of photoexcited carrier relaxation dynamics in $\mathrm{Yb}_{1-x} \mathrm{Lu}_{x} \mathrm{Al}_{3}$ system, where doping was continuously varied between $0 \leq x \leq 1$, we show that the hybridization gap persists up to the critical doping $x \approx 0.4$. This is consistent with inelastic neutron-scattering data. ${ }^{17}$ Indeed, the percolation threshold for the cubic system is $\approx 0.3{ }^{25}$ This observation implies that the Kondo lattice is a very robust feature, and suggest the Anderson lattice model to be a minimum model for the description of this class of materials.

In $\mathrm{Yb}_{1-x} \mathrm{Lu}_{x} \mathrm{Al}_{3}$, below some doping dependent temperature $T^{*}(x)$, the relaxation-time divergence due to the relaxation bottleneck is cut. This observation can be naturally explained by considering a parallel temperature independent relaxation channel. Since $\mathrm{YbAl}_{3}$ is known for its large electronic mean-free path, we consider ballistic electronic transport out of the probed volume as a possible competing process. We developed a theoretical model, describing the dynamics of excess quasiparticle density for such a case. Comparison with the experimental data showed that the functional form of relaxation below $T^{*}(x)$, the temperature dependence of relaxation time, as well as the doping dependence of the low-temperature relaxation rate in $\mathrm{Yb}_{1-x} \mathrm{Lu}_{x} \mathrm{Al}_{3}$ can be well accounted for by this model with the dominant ballistic electron transport at low $T$. The strikingly sharp anomaly in $\tau_{*}^{-1}(x)$ at $x \approx 0.1$ clearly requires further experimental and theoretical work.

\section{ACKNOWLEDGMENTS}

This work was supported by the Alexander von Humboldt Foundation, Zukunftskolleg and Center for Applied Photonics at the University of Konstanz, the Laboratory Directed Research and Development program at Los Alamos National Laboratory, and the Center for Integrated Nanotechnologies at LANL.
${ }^{1}$ A. C. Hewson, The Kondo Problem to Heavy Fermions (Cambridge University Press, Cambridge, 1993).

${ }^{2}$ L. Degiorgi, Rev. Mod. Phys. 71, 687 (1999).

${ }^{3}$ P. S. Riseborough, Adv. Phys. 49, 257 (2000).

${ }^{4}$ J. Demsar, V. K. Thorsmølle, J. L. Sarrao, and A. J. Taylor, Phys. Rev. Lett. 96, 037401 (2006).

${ }^{5}$ J. Demsar, J. L. Sarrao, and A. J. Taylor, J. Phys.: Condens. Matter 18, R281 (2006).

${ }^{6}$ J. Demsar, R. D. Averitt, K. H. Ahn, M. J. Graf, S. A. Trugman, V. V. Kabanov, J. L. Sarrao, and A. J. Taylor, Phys. Rev. Lett. 91, 027401 (2003).

${ }^{7}$ K. H. Ahn, M. J. Graf, S. A. Trugman, J. Demsar, R. D. Averitt, J. L. Sarrao, and A. J. Taylor, Phys. Rev. B 69, 045114 (2004).

${ }^{8}$ K. S. Burch, E. E. M. Chia, D. Talbayev, B. C. Sales, D. Mandrus, A. J. Taylor, and R. D. Averitt, Phys. Rev. Lett. 100, 026409 (2008).

${ }^{9}$ A. Rothwarf and B. N. Taylor, Phys. Rev. Lett. 19, 27 (1967).
${ }^{10}$ J. Demsar, R. D. Averitt, A. J. Taylor, V. V. Kabanov, W. N. Kang, H. J. Kim, E. M. Choi, and S. I. Lee, Phys. Rev. Lett. 91, 267002 (2003)

${ }^{11}$ V. V. Kabanov, J. Demsar, and D. Mihailovic, Phys. Rev. Lett. 95, 147002 (2005).

${ }^{12}$ A. L. Cornelius et al., Phys. Rev. Lett. 88, 117201 (2002).

${ }^{13}$ E. D. Bauer, C. H. Booth, J. M. Lawrence, M. F. Hundley, J. L. Sarrao, J. D. Thompson, P. S. Riseborough, and T. Ebihara, Phys. Rev. B 69, 125102 (2004).

${ }^{14}$ Fluence was kept low to avoid sample heating. Over the fluence range $F=0.1-2 \mu \mathrm{J} / \mathrm{cm}^{2}$ no nonlinearities were observed; i.e., the photoinduced transient scales with $F$.

${ }^{15}$ S. J. Lee, J. M. Park, P. C. Canfield, and D. W. Lynch, Phys. Rev. B 67, 075104 (2003).

${ }^{16}$ R. H. M. Groeneveld, R. Sprik, and A. Lagendijk, Phys. Rev. B 51, 11433 (1995), and references therein.

${ }^{17}$ Raymond Osborn, Eugene A. Goremychkin, Igor L. Sashin, and 
Amir P. Murani, J. Appl. Phys. 85, 5344 (1999).

${ }^{18}$ H. Okamura, T. Michizawa, T. Nanba, and T. Ebihari, J. Phys. Soc. Jpn. 73, 2045 (2004); H. Okamura, T. Michizawa, M. Matsunami, S. Kumura, T. Nanba, T. Ebihara, F. Iga, and T. Takabatake, J. Magn. Magn. Mater. 272-276, E51 (2004).

${ }^{19}$ T. Ebihara et al., J. Phys. Soc. Jpn. 69, 895 (2000).

${ }^{20}$ I. Sakamoto, G. F. Chen, S. Ohara, H. Harima, and S. Maruno, J. Alloys Compd. 323-324, 623 (2001).

${ }^{21}$ See, e.g., E. Knoesel, A. Hotzel, and M. Wolf, Phys. Rev. B 57, 12812 (1998).

${ }^{22}$ Analysis of the relaxation beyond the $\tau$ approximation (Ref. 26) provides about the same qualitatively picture [V. V. Kabanov et al. (unpublished)].

${ }^{23}$ Such choice of the excitation profile is used to satisfy the condition $\partial F / \partial x=0$ at $x=0$. This condition means that quasiparticles cannot escape from the sample through the surface. The results are independent of the particular choice of excitation profile.

${ }^{24}$ J. L. Sarrao et al., Phys. Rev. B 59, 6855 (1999).

${ }^{25}$ See, e.g., C. D. Lorenz and R. M. Ziff, J. Phys. A 31, 8147 (1998).

${ }^{26}$ V. V. Kabanov and A. S. Alexandrov, Phys. Rev. B 78, 174514 (2008). 